\title{
Nutrição e doença renal crônica (DRC): Apresentação das novas recomendações e padrões alimentares conforme as últimas evidências científicas
}

\author{
Nutrition and chronic kidney disease (CKD): Presentation of new recommendations and food \\ standards by the last scientific evidence \\ Nutrición y enfermedad renal crónica (ERC): Presentación de nuevas recomendaciones y normas \\ alimentarias según la última prueba científica
}

\author{
Moisés Iasley Lima Vasconcelos \\ ORCID: https://orcid.org/0000-0002-8724-1063 \\ Centro Universitário Estácio do Ceará, Brasil \\ E-mail: moisesasley@gmail.com \\ Helder Matheus Alves Fernandes \\ ORCID: https://orcid.org/0000-0003-2068-9071 \\ Faculdade Nova Esperança de Mossoró, Brasil \\ E-mail: heldermatheus10@hotmail.com \\ Elane da Silva Barbosa \\ ORCID: https://orcid.org/0000-0002-2668-8064 \\ Universidade Estadual do Ceará, Brasil \\ E-mail: elanesilvabarbosa@hotmail.com \\ Richardeson Fagner de Oliveira Grangeiro \\ ORCID: https://orcid.org/0000-0001-6760-267X \\ Universidade do Estado do Rio Grande do Norte, Brasil \\ E-mail: richardesonfagner@ hotmail.com \\ Daniel Barbosa Gregório de Sena \\ ORCID: https://orcid.org/0000-0003-1105-6731 \\ Faculdade Nova Esperança de Mossoró, Brasil \\ E-mail: danielnutriacademico@gmail.com \\ Vitória Celeste Barbosa Lopes \\ ORCID: https://orcid.org/0000-0002-5448-2186 \\ Faculdade Nova Esperança de Mossoró, Brasil \\ E-mail: vitoriacelestebl@gmail.com \\ Mariane de Oliveira Sandes \\ ORCID: https://orcid.org/0000-0002-3757-7423 \\ Centro Universitário Estácio do Ceará, Brasil \\ E-mail: mariane.sandes@outlook.com \\ Lorena Santiago de Sousa \\ ORCID: https://orcid.org/0000-0002-7530-1671 \\ Faculdade Nova Esperança de Mossoró, Brasil \\ E-mail: lorenass010399@gmail.com \\ Renato Gondim de Oliveira \\ ORCID: https://orcid.org/0000-0001-7894-2418 \\ Universidade Potiguar, Brasil \\ E-mail: renatogondm@gmail.com \\ Viviane Lopes Tabosa \\ ORCID: https://orcid.org/0000-0003-1018-4440 \\ Universidade Estadual do Ceará, Brasil \\ E-mail: vivianetabosa@hotmail.com \\ Luisa Ariel Rodrigues Gomes Firmino \\ ORCID: https://orcid.org/0000-0001-9424-2411 \\ Centro Universitário Estácio do Ceará, Brasil \\ E-mail: luisaarielgomes@gmail.com
}

\section{Resumo}

A doença renal crônica (DRC) é uma síndrome lenta, silenciosa, irreversível e progressiva da função renal e, assim, o manejo nutricional permanece entre as intervenções mais importantes para retardar a progressão da DRC. Trata-se de Revisão Sistemática, realizada nas bases de dados: LILACS, SciELO e ScienceDirect, a partir dos seguintes descritores: Nutrição, Doença Renal Crônica, Padrão Alimentar e Dieta, na qual foram utilizados os operadores 
booleanos "AND" e "OR", realizando as distintas combinações. Compuseram o corpus desta investigação 18 artigos científicos, incluindo duas diretrizes publicadas nos últimos cinco anos. A fim de apresentar de forma sistemática as evidências reunidas em torno das novas recomendações de nutrição e DRC, foram elaborados cinco quadros contendo as informações sobre as publicações, estágios da doença renal, recomendações de proteínas no tratamento conservador e na terapia renal substitutiva, de carboidratos, lipídeos e energia em ambas modalidades. Identifica-se que as evidências em torno de um padrão alimentar ainda são bastante controversas, no entanto a literatura apresenta que o padrão dietético com enfoque em alimentos de origem vegetal, frutas, grãos integrais, leguminosas, rico em fibras e consumo moderado de alimentos de origem animal, promovem efeitos benefícios no gerenciamento e na prevenção das complicações metabólicas da DRC. Portanto, até o momento não existe um padrão específico para controlar os distúrbios das nefropatias, visto que o controle dietético vai da individualidade de cada paciente e análise investigativa-crítica do nutricionista.

Palavras-chave: Nutrição; Doença renal crônica; Padrão alimentar; Dieta.

\begin{abstract}
Chronic kidney disease (CKD) is a slow, silent, irreversible and progressive syndrome of kidney function and, thus, nutritional management remains among the most important interventions to slow the progression of CKD. This is a systematic review, carried out on the databases: LILACS, SciELO and ScienceDirect, based on the following descriptors: Nutrition, Chronic Kidney Disease, Food Pattern and Diet, in which the Boolean operators "AND" and "OR" were used, carrying out the different combinations. The corpus of this investigation comprised 18 scientific articles, including two guidelines published in the last five years. In order to systematically present the evidence gathered around the new nutrition and CKD recommendations, five tables were prepared containing information about publications, stages of kidney disease, protein recommendations in conservative treatment and in renal replacement therapy, carbohydrates, lipids and energy in both modalities. It is identified that the evidence regarding a dietary pattern is still quite controversial, however the literature shows that the dietary pattern with a focus on foods of plant origin, fruits, whole grains, legumes, rich in fiber and moderate consumption of foods animal origin, promote beneficial effects in the management and prevention of metabolic complications of CKD. Therefore, to date, there is no specific standard for controlling nephropathy disorders, since dietary control goes from the individuality of each patient and the investigative-critical analysis of the nutritionist.
\end{abstract}

Keywords: Nutrition; Chronic kidney disease; Food standard; Diet.

\title{
Resumen
}

La enfermedad renal crónica (ERC) es un síndrome de la función renal lento, silencioso, irreversible y progresivo, por lo que el manejo nutricional sigue siendo una de las intervenciones más importantes para frenar la progresión de la ERC. Se trata de una revisión sistemática, realizada sobre las bases de datos: LILACS, SciELO y ScienceDirect, basada en los siguientes descriptores: Nutrición, Enfermedad Renal Crónica, Patrón Alimentario y Dieta, en la que se utilizaron los operadores booleanos "Y" y "O", realizando las diferentes combinaciones. El corpus de esta investigación estuvo compuesto por 18 artículos científicos, incluyendo dos guías publicadas en los últimos cinco años. Con el fin de presentar sistemáticamente la evidencia recogida en torno a las nuevas recomendaciones nutricionales y de ERC, se elaboraron cinco tablas que contienen información sobre publicaciones, estadios de la enfermedad renal, recomendaciones proteicas en tratamiento conservador y en terapia renal sustitutiva, carbohidratos, lípidos y energía en ambas modalidades. Se identifica que la evidencia respecto a un patrón dietético aún es bastante controvertida, sin embargo la literatura muestra que el patrón dietético con enfoque en alimentos de origen vegetal, frutas, cereales integrales, legumbres, ricos en fibra y consumo moderado de alimentos de origen animal, promover efectos beneficiosos en el manejo y prevención de las complicaciones metabólicas de la ERC. Por tanto, a la fecha, no existe un estándar específico para el control de los trastornos nefropatológicos, ya que el control dietético pasa por la individualidad de cada paciente y el análisis investigativo-crítico del nutricionista.

Palabras clave: Nutrición; Enfermedad renal crónica; Norma alimentaria; Dieta.

\section{Introdução}

A prática clínica baseada em evidências leva em consideração os conhecimentos explícitos e subjacentes, entendendo que é possível colocar em prática as competências e habilidades profissionais relacionadas às recomendações clínicas. No entanto, a dúvida passa a fazer parte do processo de decisão, visto que algumas recomendações são tão complexas que só se consegue visualizar através da individualidade de cada paciente, principalmente, nas recomendações para pacientes renais crônicos, sendo necessária uma habilidade técnica relativa a saber/fazer que pondere sobre as recomendações e a história clínica do paciente (Joshi, Mcmacken \& Zadeh, 2020). 
Partindo dessa contextualização, a Doença Renal Crônica (DRC) pode ser caracterizada como uma síndrome, a qual ocasiona uma perda lenta, silenciosa e progressiva da função renal. Por definição, é considerada quando existem alterações renais, sejam elas estruturais ou funcionais, com duração superior a três meses e/ou uma diminuição da taxa de filtração glomerular (TFG) para valores menores do que $60 \mathrm{ml} / \mathrm{min} / 1,73 \mathrm{~m}^{2}$, também por um período maior do que três meses (Cuppari, 2019). Assim, uma das funções do sistema renal consiste na produção de eritropoietina, manutenção do equilíbrio hidroeletrolítico, solutos orgânicos e ácido-base, ativação dos mecanismos renina-angiotensina e ativação da vitamina D (Krause, Mahan \& Escott-Stump, 2018).

Inclusive um dos grandes fatores relacionados à causa de mortalidade devido a DRC é o aumento da incidência de morbidade e mortalidade por doenças cardiovasculares (DCV), principalmente, diabetes mellitus (DM) e hipertensão arterial sistêmica (HAS), que podem estar coexistindo no percurso da DRC, indicando um contínuo desafio de saúde que precisam ser enfrentadas com adoção de reformulação das políticas públicas e medidas preventivas (Goraya \& Wesson, 2015).

Nesse panorama, o papel de um padrão alimentar saudável é muito mais complexo do que a ingestão e/ou as combinações de nutrientes. O manejo alimentar tradicional da DRC se concentra predominantemente na quantidade da dieta ao invés da sua qualidade. Com base nesse pressuposto, são necessárias modificações para tornar a dieta do tipo "Plant Based" isto é, baseada em vegetais, Estilo Mediterrâneo, vegetariano e Dietary Approach to Stop Hypertension (DASH), realista em pacientes com DRC, a fim de atender às necessidades individuais prescritos em uma restrição específica de potássio, sódio, líquidos e fósforo (Chauveau et al., 2017).

Nesse sentido, até 20 anos atrás, acreditava-se que pacientes renais não poderiam comer vegetais, frutas, legumes e hortaliças por conta da hipercalemia, isto é, quando os níveis de potássio estão aumentando, podendo causar arritmia cardíaca e levar à morte, assim como alimentos de origem animal, por conta do fósforo, mas isso foi se desconfigurando no decorrer do tempo e surgindo novos padrões alimentares que atendem aos objetivos dos pacientes, a partir da diminuição dos sintomas, o que contribui para o retardo do comprometimento da função renal (Serdan et al., 2020).

Logo, dietas baseadas em vegetais, frutas, fibras, hortaliças, grãos, leguminosas exercem efeitos metabólicos benéficos em pacientes com DRC não diabética, e parecem ser seguros, embora esses dados tenham sido obtidos a partir de estudos ainda iniciais (Maggo, 2017; Jain \& Reilly, 2014). Ainda há evidências limitadas que confirmam a eficácia, a segurança da intervenção, em particular a longo prazo, de adição de suplementos de cetoanálogo (SC) a uma dieta baixa em proteínas (DBP) no planejamento dietético, uma vez que os cetoácidos, além de serem caro na aquisição, nem todo paciente vai ter condição de comprar. Por isso que os primeiros protocolos adotados nos pacientes renais é a restrição proteica, principalmente, restringindo grandes grupos alimentares que são importantes para alimentação saudável (Jain \& Reilly, 2014).

Contextualizando historicamente essa restrição, os primeiros protocolos de restrição proteica, em especial, no tratamento conservador, a etapa anterior do tratamento dialítico, surgiram no final da década de 1800, quando os pesquisadores descobriram que a restrição de proteínas melhorou os sinais e sintomas de uremia no intuito de retardar o máximo possível o início do tratamento dialítico. Até hoje, essas medidas terapêuticas vêm sendo adotadas na prática clínica, sendo necessário, antes de tudo, avaliar a condição o paciente, qual estágio de insuficiência renal o mesmo apresenta e se realmente é necessário, visto que isso influencia nas escolhas alimentares e na aquisição dos mesmos (Messa, 2020).

Nessa perspectiva, surgiu uma nova atualização da diretriz de nutrição para adultos com DRC publicada pela National Kidney Foundation (NKF) em parceria com The Academy of Nutrition and Dietetics (AND) no final do segundo semestre de 2020, com intuito de reformular as práticas e protocolos das condutas dietoterápicas dos nutricionistas para permitir segurança nos atendimentos e melhorar a conduta conforme cada individualidade do pacientes. A diretriz se tornou um marco muito importante no mundo da nefrologia, uma vez que, fazia 20 anos que ela não tinha sido atualizada e trouxe questionamento 
bastante poder antes que se tinha atualmente sobre a dieta dos nefropatia e novas recomendações nutricionais (Kdoqi, 2020; Kdigo, 2020).

Portanto, o objetivo do presente estudo é apresentar as principais recomendações nutricionais e padrões dietéticos analisadas pela literatura no tratamento em pacientes com doença renal crônica (DRC).

\section{Metodologia}

Trata-se de revisão sistemática, em que visa reunir as pesquisas disponíveis sobre determinada temática e direcionar as evidências, fundamentando-se em conhecimento científico. Assim, este tipo de pesquisa visa reduzir o viés através do uso de métodos explícitos, sendo sua investigação bem definida, delineada, transparente e reaplicável, avaliando de forma crítica, ordenada e abrangente os achados dos estudos de forma organizada (Donato \& Donato, 2019).

Nesse sentido, foram definidos como critérios de inclusão: Artigos na íntegra online, em inglês, do tipo transversal e ensaios clínicos randomizado, visto que há carências em estudos nacionais, publicados em revistas indexadas, nos últimos cinco anos, discorrendo acerca das atualizações da assistência nutricional que a literatura aponta no manejo em pacientes com DRC para o público que está no processo de tratamento conservador ou terapia renal substitutiva (TRS), por intermédio da diálise, ou seja, hemodiálise (HD) ou diálise peritoneal (DP) e transplante renal (TxR). Os critérios de exclusão, por sua vez, foram: estudos de revisões, relatos de experiências, com crianças e/ou adolescentes, e adultos com insuficiência aguda, Nefrolitíase (cálculo renal) e síndrome renal policística (SRP).

Somando-se a isso, destaca-se que, em decorrência da escassez de artigos que tratem sobre essa temática, conforme os critérios previamente elencados acima, a título de complementação da bibliografia, foram utilizadas duas diretrizes clínicas para dar mais embasamento científico na produção, visto que as diretrizes clínicas servem para nortear a prática clínica do profissional. A diretriz é, então, um dos principais documentos que reúne evidências científicas que permitem uma melhor conduta terapêutica conforme as investigações mais recentes em relação, no caso deste estudo, à doença renal

A busca ocorreu por meio de três bases de dados: Literatura Latino-Americana e do Caribe em Ciências da Saúde LILACS, Scientific Electronic Library Online - SciELO e ScienceDirect, utilizando-se os seguintes descritores, padronizados e disponíveis nos descritores em Ciências da Saúde - DeCS: "Nutrição", "Doença Renal Crônica", "Padrão Alimentar;" e "Dieta." e Medical Subject Headings (MeSH): "Nutrition", "Chronic Kidney Disease", "Food Standard;" e "Diet." utilizando os operadores booleanos "AND" e "OR" e realizando as distintas combinações/cruzamentos nas bases de dados.

A escolha dessas bases de dados ocorreu pelo fato de terem maior visibilidade científica na área de nutrição e DRC que abrange a assistência nutricional, protocolos, atualizações e orientações que permitem ao nutricionista conduzir o paciente com segurança em frente com essa comorbidade.

O levantamento realizou-se de vinte de janeiro de 2021 a dezesseis de fevereiro de 2021, sendo feita leitura prévia dos títulos e resumos dos estudos, e caso tivesse dúvida quanto à sua adequação, era realizada a leitura do artigo completo. Então, a amostra constituiu 16 artigos, além de duas diretrizes clínicas atualizadas no ano de 2020, perfazendo um total de 18 produções científicas para construção deste artigo.

\section{Resultados}

Conforme o mapeamento dos artigos, obtiveram-se na LILACS, 83 artigos; na ScienceDirect, 322 artigos; e por fim, na Scielo, 211 artigos, perfazendo um total de 616 artigos encontrados conforme os distintos cruzamentos entre os descritores. Para tanto, após análise, seleção, elegibilidade e leitura na íntegra dos artigos, foram selecionados 16 artigos elegíveis para compor o corpus da pesquisa, além disso, foram incluídas duas diretrizes clínicas, totalizando 18 documentos científicos. 
No que concerne ao ano de publicação, quatro artigos foram publicados entre 2017 e 2019; três, no ano de 2018; seis no ano de 2020; e apenas um, no ano de 2016. O maior quantitativo de artigos no de 2020 se deu por justificativa que no respectivo ano, ocorreu atualização da nutrição e DRC por meio das duas diretrizes clínicas, que instigaram os pesquisadores a repensar e analisar como essas novas recomendações, sugestões e análises das diretrizes podem estar repercutindo atualmente com o paciente, sobretudo, naqueles em estágios mais avançados e que realizam terapia dialítica.

Portanto, mediante os resultados do presente estudo, foi elaborado um quadro, o qual apresenta os artigos de acordo com o ano, bem como os autores, título, periódico e objetivo do estudo.

Quadro 1 - Estudos incluídos na revisão sistemática em relação ao ano de publicação, aos autores, título do artigo, indexação periódica e objetivo.

\begin{tabular}{|c|c|c|c|c|c|}
\hline$N^{\circ}$ & ANO & AUTORES & TÍTULO & PERIÓDICO & OBJETIVO \\
\hline 01 & 2016 & $\begin{array}{c}\text { Banerjee, T.; Liu, Y.; Crews, } \\
\text { D.C. }\end{array}$ & $\begin{array}{c}\text { Dietary Patterns and CKD } \\
\text { Progression }\end{array}$ & Blood Purif & $\begin{array}{l}\text { Avaliar os diferentes Padrões dietéticos } \\
\text { e progressão da DRC. }\end{array}$ \\
\hline 02 & 2017 & Kammoun, K. et al., & $\begin{array}{l}\text { Diet in chronic kidney } \\
\text { disease in a } \\
\text { Mediterranean African } \\
\text { country }\end{array}$ & BMC Nephrology & $\begin{array}{l}\text { Analisar a dieta na doença renal crônica } \\
\text { em um País africano mediterrâneo }\end{array}$ \\
\hline
\end{tabular}

$2017 \quad$ Cupisti, A. et al., $\quad$ F

Non-Traditional Aspects of Renal Diets: Focus on Fiber, Alkali and Vitamin K1 Intake
Analisa o conteúdo de vitamina K1, Nutrients álcali e fibra de diferentes dietas renais utilizadas em pacientes com DRC prédiálise, e trata da importância desses nutrientes na dieta alimentar gestão de DRC.
Rysz, J. et al.,
The Effect of Diet on the Survival of Patients with Chronic Kidney Disease
Nutrients

Vegetarian Diet in Chronic Kidney Disease A Friend or Foe
Nutrients

Revisar os resultados de estudos sobre o impacto da dieta na sobrevida de pacientes com DRC.

Analisar dieta vegetariana e suas modificações são adequadas para doenças crônicas pacientes com doença renal.

Clegg, D.J.; Kathleen, M.; Gallant, $\mathrm{H}$.

\section{Adequacy of Plant-Based Proteins in Chronic Kidney Disease}

\section{Nutritional}

Considerations for Dialysis Vegetarian Patients
Journal of Renal

Nutrition

Journal of Renal Nutrition
Analisar adequação de proteínas baseadas em plantas em Doença Renal Crônica. 


\begin{tabular}{|c|c|c|c|c|c|}
\hline 09 & 2019 & Kim, H. et al., & $\begin{array}{l}\text { Plant-Based Diets and } \\
\text { Incident CKD and Kidney } \\
\text { Function }\end{array}$ & $\begin{array}{l}\text { Clinical Journal of } \\
\text { the American } \\
\text { Society of } \\
\text { Nephrology }\end{array}$ & $\begin{array}{l}\text { Investigar prospectivamente a relação } \\
\text { em um estudo de base populacional e } \\
\text { avaliar se o risco variou por diferentes } \\
\text { tipos de dietas baseadas em plantas. }\end{array}$ \\
\hline 10 & 2019 & Chauveau, P. et al., & $\begin{array}{l}\text { Vegetarian diets and } \\
\text { chronic kidney disease }\end{array}$ & $\begin{array}{l}\text { Nephrol Dial } \\
\text { Transplant }\end{array}$ & $\begin{array}{l}\text { Associar as contribuições da Dietas } \\
\text { vegetarianas e doença renal crônica. }\end{array}$ \\
\hline 12 & 2019 & Liu, H.W et al., & $\begin{array}{c}\text { Association of Vegetarian } \\
\text { Diet with Chronic Kidney } \\
\text { Disease }\end{array}$ & Nutrients & $\begin{array}{l}\text { Avaliar a associação entre dietas } \\
\text { vegetarianas e CKD. Um estudo } \\
\text { transversal foi baseado em indivíduos } \\
\text { que receberam exames no Hospital } \\
\text { Taipei Tzu Chi de } 5 \text { de setembro de } \\
2005 \text { a } 31 \text { de dezembro de } 2016 \text {. }\end{array}$ \\
\hline 13 & 2020 & Lanuza, F. et al., & $\begin{array}{l}\text { Wholegrain Consumption } \\
\text { and Risk Factors for } \\
\text { Cardiorenal Metabolic } \\
\text { Diseases in Chile }\end{array}$ & Nutrients & $\begin{array}{l}\text { Avaliar a associação entre consumo do } \\
\text { GT e fatores de risco para CRMD na } \\
\text { Pesquisa Nacional de Saúde do Chile } \\
\text { 2016-2017. }\end{array}$ \\
\hline 14 & 2020 & Letourneau, P. et al., & $\begin{array}{l}\text { Source and Composition } \\
\text { in Amino Acid of Dietary } \\
\text { Proteins in the Primary } \\
\text { Prevention and Treatment } \\
\text { of CKD }\end{array}$ & Nutrients & $\begin{array}{l}\text { Revisar as evidências recentes } \\
\text { sugerindo que a fonte de proteína } \\
\text { (animal versus planta) e a composição } \\
\text { de AAs afetam especificamente a } \\
\text { progressão da DRC e complicações. }\end{array}$ \\
\hline 16 & 2020 & Ramos, C.I et al., & $\begin{array}{l}\text { Does dietary potassium } \\
\text { intake associate with } \\
\text { hyperkalemia in patients } \\
\text { with chronic kidney } \\
\text { disease? }\end{array}$ & $\begin{array}{l}\text { Nephrol Dial } \\
\text { Transplant }\end{array}$ & $\begin{array}{l}\text { Investigar se o potássio da dieta ou a } \\
\text { ingestão de determinados grupos de } \\
\text { alimentos se associa ao potássio sérico } \\
\text { frente a outros fatores de risco. }\end{array}$ \\
\hline 17 & 2020 & Kidney International & $\begin{array}{l}\text { KDIGO } 2020 \text { Clinical } \\
\text { Practice Guideline for } \\
\text { Diabetes Management in } \\
\text { Chronic Kidney Disease }\end{array}$ & $\begin{array}{c}\text { Kidney } \\
\text { International }\end{array}$ & $\begin{array}{l}\text { O objetivo da diretriz é gerar um } \\
\text { recurso útil para os médicos e } \\
\text { pacientes, fornecendo recomendações } \\
\text { acionáveis com infográficos baseados } \\
\text { em um rigoroso, revisão sistemática } \\
\text { formal da literatura }\end{array}$ \\
\hline 18 & 2020 & Ikizler et al. & $\begin{array}{l}\text { Diretriz de Prática Clínica } \\
\text { KDOQI para Nutrição na } \\
\text { DRC: Atualização de } \\
2020\end{array}$ & $\begin{array}{c}\text { American Journal of } \\
\text { Kidney Diseases }\end{array}$ & $\begin{array}{l}\text { O objetivo da diretriz é fornecer } \\
\text { informações atualizadas e abrangentes } \\
\text { sobre a compreensão e o cuidado de } \\
\text { pacientes com doença renal crônica } \\
\text { (DRC), especialmente em termos de seu } \\
\text { meio metabólico e nutricional, para o } \\
\text { clínico em exercício e profissionais de } \\
\text { saúde associados. }\end{array}$ \\
\hline
\end{tabular}

Fonte: Elaborado pelos autores (2021). 
Para apresentar sistematicamente os resultados da presente investigação, foram elaborados quadros $02,03,04$ e 05 contendo as novas atualizações por intermédio das diretrizes clínica das novas classificações da TFG utilizada para Classificar a DRC, bem como em relação às recomendações de proteínas, energia, carboidratos e lipídeos no tratamento conservador e tratamento dialítico. A seguir, será apresentada cada uma delas:

Quadro 2 - Estágio da Doença Renal Crônica (DRC) conforme a Taxa de Filtração Glomerular (TFG) na presença ou ausência de Albuminúria, adaptadas das diretrizes clínicas, publicadas em 2020.

Estágios da doença renal crônica

\begin{tabular}{|c|c|c|}
\hline Estágios & $\begin{array}{c}\text { Taxa De Filtração Glomerular } \\
\left(\left(\mathrm{ml} / \mathbf{m i n} / 1.73 m^{3}\right)\right.\end{array}$ & Albuminúria \\
\hline 1 & $\geq 90$ & Presente \\
\hline 2 & $60-89$ & Presente \\
\hline $\mathbf{3 A}$ & $45-59$ & Presente ou ausente \\
\hline 3B & $30-44$ & Presente ou ausente \\
\hline 4 & $15-29$ & Presente ou ausente \\
\hline 5 & $<15$ & Presente ou ausente \\
\hline
\end{tabular}

Fonte: Elaborado pelos autores (2021).

Os estágios 1-5 são do tratamento conservador, e a partir do estágio 5 entra no tratamento para TRS, caracterizado por TxE, HD e DP. A seguir serão apresentadas as recomendações de proteínas no tratamento conservador, vale destacar que, a média de consumo no Brasil de proteínas é de 1,3g/kg/dia e também, essas recomendações são para metabolicamente estáveis, sem Diabetes e sob supervisão clínica (Kdoqi, 2020).

Metabolicamente estável é caracterizada pela ausência de Doença inflamatória ou infecciosa ativa, ocasionada por Hospitalização recente, Diabetes descompensado, diminuição da perda de peso significativa e recente, além de Câncer (Kdoqi, 2020).

Quadro 3 - Recomendações de proteínas no tratamento conservador, conforme os estágios da TFG e a classificação do planejamento dietético, adaptadas nas diretrizes clínicas, publicadas em 2020.

\begin{tabular}{|c|c|c|}
\hline Estágios & Recomendação & Classificação \\
\hline 1 e 2 & Normal - 0,8 a $1 \mathrm{~g} / \mathrm{kg} / \mathrm{dia}$ & Normoproteica \\
\hline 3 a 5, sem Diabetes & $\begin{array}{c}0,55 \text { a } 0,6 \mathrm{~g} / \mathrm{kg} / \mathrm{dia} \text { ou } 0,28 \mathrm{a} \\
0,43 \mathrm{~g} / \mathrm{kg} / \mathrm{dia}+ \\
\text { cetoácidos/análogos de } \\
\text { aminoácidos essenciais. }\end{array}$ & Hipoproteica \\
\hline 3 a 5, com Diabetes & 0,6 a $0,8 \mathrm{~g} / \mathrm{kg} / \mathrm{dia}$ & Hipoproteica a normoproteica \\
\hline
\end{tabular}

Fonte: Elaborado pelos autores (2021). 
A seguir, serão apresentadas as recomendações de proteínas no tratamento dialítico, nesta instância, essas recomendações seguem o mesmo protocolo de estabilidade no tratamento conservador, ou seja, precisam ser metabolicamente estáveis e precisa avaliar individualmente, a fim de auxiliar no controle glicêmico (Kdoqi, 2020).

Nesse sentido, as recomendações proteicas são maiores na diálise por conta que, no processo dialítico, ocorrem perdas de proteínas de 1 a $3 \mathrm{~g} /$ sessão e de aminoácidos e peptídeos 10 a 12g/sessão na HD, já na DP a perda é bem maior, de 5 a $15 \mathrm{~g} /$ sessão de proteínas e $3 \mathrm{~g} / \mathrm{sessão} \mathrm{de} \mathrm{aminoácidos} \mathrm{e} \mathrm{peptídeos,} \mathrm{sendo} \mathrm{dessas} \mathrm{proteínas,} \mathrm{uma} \mathrm{perda} \mathrm{de} \mathrm{50-70 \%} \mathrm{albumina}$ (KDOQI, 2020).

Quadro 4 - Recomendações de proteínas na Terapia Renal Substitutiva (TRS) com ou sem diabetes, recomendação e classificação do planejamento dietético, adaptadas das diretrizes clínicas, publicadas em 2020.

\section{Recomendações de proteína no tratamento dialítico}

HD e DP, sem Diabetes

HD e DP, com Diabetes
1,0 a $1,2 \mathrm{~g} / \mathrm{kg} / \mathrm{dia}$

Pacientes com risco de

hipoglicemia ou hiperglicemia,

níveis maiores de proteína

podem ser considerados.
Normoproteica a hiperproteica

Normoproteica a hiperproteica

Fonte: Elaborado pelos autores (2021).

A seguir, dispõem-se as recomendações de energia, carboidratos e lipídeos, no tratamento conservador e TRS. Logo, a recomendação está abrangendo apenas pacientes metabolicamente estáveis. Além disso, a escolha calórica deve ser baseada na idade, sexo, nível de atividade física, composição corporal, perda ou ganho de peso, estágio da DRC e comorbidades ou presença de inflamação (Kdoqi, 2020).

Quadro 5 - Recomendações de energia, carboidratos e lipídeos no tratamento conservador (TC) e na Terapia Renal Substitutiva (TRS), adaptadas das diretrizes clínicas, publicadas em 2020.

\section{Recomendações de nutrientes em TC e TRS}

\begin{tabular}{cc} 
Estágio & Recomendação \\
\hline $\mathbf{1}$ a $\mathbf{5}$ & 25 a $35 \mathrm{kcal} / \mathrm{kg} / \mathrm{dia}$ \\
\hline Carboidratos & 50 a $60 \%$ do VET \\
\hline Lipídeos & 25 a $35 \%$ do VET \\
\hline
\end{tabular}

Evitar prescrever $<25 \mathrm{kcal} / \mathrm{kg} / \mathrm{dia}$

$\rightarrow$ Garantir balanço nitrogenado

Valor Calórico Total - VET

Fonte: Elaborado pelos autores (2021).

Com isso, um dos alimentos excelentes com grande qualidade nutricional para o aumento do aporte calórico sem alterar nas proteínas tratam-se dos carboidratos e lipídeos, que podem ser encontrados em raízes, tubérculos, farináceos, mel e óleos/azeites como estratégia do aporte calórico.

\section{Discussões}

Os hábitos alimentares ocidentais são excessivamente ricos em proteínas, gordura saturada e sódio, e pobres em frutas, vegetais, grãos e fibras. Considerando as recomendações da Organização Mundial da Saúde (OMS) para população 
geral, a mesma preconiza um consumo ideal em torno de $0,83 \mathrm{~g}$ de proteínas $/ \mathrm{kg} / \mathrm{dia}$, no entanto o consumo é bem superior do que o recomendado, mas, surpreendentemente, quando faz um paralelo entre o consumo de proteínas para o público geral e para os nefropatas, acaba não sendo uma restrição severa, e sim uma adequação (Cupisti et al., 2017).

Para tanto, as novas evidências demonstram que adotar um padrão alimentar saudável no estilo DASH, Vegetariano, Baseado em Vegetais "Plant Based" e/ou mediterrâneas, podem se tornar úteis como estratégia de prevenção para diminuir a prevalência de insuficiência renal, sobretudo na Plant Based, que está associado a uma menor ingestão de energia e proteína do que as dietas onívoras, sendo compatível com as recomendações nutricionais para insuficiência renal crônica (IRC) (Chauveau et al 2019; Carrero et al., 2020).

A dieta Mediterrânea caracteriza-se por ser rica em frutas, hortaliças, cereais integrais, incluindo as categorias de grãos e leguminosas, além de quantidades modestas de azeite de oliva (rica em gordura monoinsaturada) com inclusão do consumo de peixes, oleaginosas, além da ingestão moderada de vinho. Além do mais, esta dieta está associada a uma menor incidência de DRC e à diminuição das complicações, entre elas se destacam: acidose metabólica e redução das toxinas urêmicas, o que contribui para amenizar os sintomas de uremia, complicações muito comuns nos renais crônicos (Kammoun, et al., 2017; Kim et al.,2019).

Logo, a Plant based é definida como o consumo de frutas, vegetais, nozes, sementes, óleos (azeite de oliva) rica em gordura monoinsaturadas, grãos integrais, legumes e leguminosas, com redução moderada do consumo de carne e laticínios entre 2 a 3 vezes por semana. Aparentemente, este padrão alimentar está repercutindo nos últimos tempos, sendo o um dos mais comentados no meio científico (Cases et al., 2019).

Destaca-se que com adesão desta dieta, houve o declínio mais lento da função renal, ou seja, da TFG, e foram apresentados protocolos de medidas preventivas quando associadas ao peso saudável dentro da normalidade. Essas influências ocorrem devido à baixa carga ácida e maior ingestão de fibras e micronutrientes, uma vez que as fibras demonstraram influenciar beneficamente no controle glicêmico e na secreção de insulina, fatores estes associados a um menor risco de microalbuminúria e proteinúria, marcadores de lesão renal (Kim et al.,2019; Clegg \& Gallant, 2019).

A dieta DASH, enfatiza o consumo também de frutas, hortaliças e laticínios com baixo teor de gordura (desnatado e semidesnatado) com inclusão de cereais integrais, frango, peixe e oleaginosas. A mesma ainda preconiza a redução do consumo de carne vermelha, doces e bebidas açucaradas, sendo, dessa forma, rica em potássio, cálcio, magnésio e fibras, e contém quantidades reduzidas de colesterol, gordura total e saturada (Lanuza et al., 2020).

Esse padrão alimentar apresenta algumas controvérsias, pois, em alguns estudos, demonstrou declínio mais rápido da TFG (Kim et al., 2019); entretanto em outros estudos, obteve-se uma diminuição da carga de ácido dietético, a qual repercute consequentemente na diminuição da progressão da doença renal. Nesse sentido, por se tratar de estudos controversos em torno desse padrão dietético, delineia-se a necessidade de realizar mais pesquisas antes da recomendação desse padrão alimentar como medida preventiva da DRC (Brzózka, Franczyk \& Rysz 2017).

Por décadas, o vegetarianismo e a doença renal foram considerados associações impossíveis de serem realizadas. Isso foi justificado por uma ideologia que as dietas à base de plantas, apesar de conterem baixas quantidades de proteínas, também são ricas em potássio e fósforo, podendo culminar na progressão da hipercalemia e hiperfosfatemia, complicações severas que podem afetar todo o estado de saúde do paciente. Porém, com o surgimento de novas pesquisas e ensaios clínicos, adoção de uma dieta vegetariana demonstra mais benefícios do que malefícios, comparados àqueles que adotam uma dieta onívora (Brzózka, Franczyk \& Rysz 2017).

O impacto de uma dieta vegetariana está associado aos benefícios cardiovasculares e à correção da diminuição de complicações que acompanham a DRC. As vantagens da adesão a esta dieta relacionam-se, principalmente, à sua composição, 
isto é, as fibras, ômega 6, potássio, magnésio, cálcio, vitamina E, vitamina C e carotenóides, bem como o muitos fitoquímicos contidos, que podem contribuir na diminuição da proteinúria, retardo da TFG, melhora na acidose metabólica e prevenção a hipercalemia (Rysz et al., 2017; Sparks, 2017).

Realizando esse paralelo entre os quatro tipos de padrão alimentar discutidos na área da nutrição e DRC, deve-se apontar que a dieta onívora que até pouco tempo era utilizada é preconizada para a DRC, tem enfrentado bastantes questionamentos, dado que as proteínas de origem animal, além de conter alto teor de gordura saturada, podem colaborar para o controle da dislipidemia e do colesterol alto, e fator de progressão da DRC. Ademais, possui também uma alta carga ácida, que contribui para a acidose metabólica; alto teor de fósforo, contribuindo para o aumento da hiperfosfatemia e piorando o estado nutricional e balanço de nutrientes, que culmina na progressão da DRC e piorar o hiperparatireoidismo secundário com efeitos deletérios na saúde óssea (Joshi, Shah \& Zadeh, 2018; Banerjee, Liu \& Crews, 2016).

Por esse ângulo, o medo de não adotar uma rica em vegetais, grãos, frutas e fibras, ocorria por causa do potássio sérico, que desencadearia a hipercalemia, geralmente acima de $5,5 \mathrm{mmol} / \mathrm{L}(\mathrm{mEq} / \mathrm{L})$, na qual o sujeito têm sintomas de convulsão, palpitação, fraqueza muscular e até mesmo a morte. Logo, com o surgimento de alguns estudos, essa percepção se torna errônea, dado que potássio dietético não foi associado ao potássio sérico ou hipercalemia em pacientes com hemodiálise (Ramos et al., 2020), inclusive, pode até favorecer a sua prevenção, visto que a dieta rica em fibras aumenta o trânsito intestinal que facilita a maior eliminação do potássio diminuindo a hipercalemia (Letourneau et al., 2020).

Nessa perspectiva, adotar um desses estilos de padrão alimentar favorece em inúmeros benefícios como medida preventiva de desenvolver DRC ou até mesmo, para pacientes com DRC, uma vez que, quando ocorre a diminuição da ingestão de proteínas de origem animal, consequentemente, ocorre a diminuição de geração de ácido, tornando-o uma dieta alcalina que melhora acidose metabólica (uma das principais causas do declínio da função renal), além de melhorar o fósforo sérico, já que é menos Biodisponivel e está na forma de ácido fítico, diminui a mortalidade do efeito significativo na proteinúria. E, por fim, ocorre o estabelecimento do equilíbrio da microbiota, visto que as fibras (prebióticos) exercem efeito no Intestino que estimulam a produção dos ácidos graxos de cadeia curta (AGG), com efeito benéfico no microbioma intestinal (LIU et al., 2019 \& Letourneau et al., 2020).

Para tanto, as últimas evidências fazem uma alerta que a troca de dietas devem ser cuidadosamente consultadas por um Nutricionista conforme a investigação da individualidade, crenças e objetivo de cada situação e/ou complicação de progressão da DRC nos pacientes, para que sejam evitados erros de uma prescrição inadequado e possibilita uma aceleração na deterioração da função renal, impactando consequentemente, na qualidade de vida do sujeito (Sparks, 2017).

\section{Considerações Finais}

Independentemente do padrão alimentar adotado, isto é, DASH, Plant Based, Vegetariano e/ou mediterrâneo, o modelo baseado em vegetais, hortaliças, frutas, grãos integrais, leguminosas, rico em fibras e consumo moderado de alimentos de origem animal, promovem efeitos benefícios adicionais no gerenciamento e na prevenção das complicações metabólicas da DRC, o que pode resultar na diminuição dos riscos relacionados a doenças vasculares, câncer e morte.

São necessários mais estudos prospectivos com maiores públicos para confirmação de qual padrão alimentar é ideal para os pacientes renais, visto que ainda existem confusão residual, divergência de estudos, variáveis questionáveis para adoção do plano. Vale ressaltar que o consumo moderado de proteína animal pode ser recomendado, visto que fornecerá aminoácidos essenciais para o reparo de tecidos, síntese de hormônios sem induzir fósforo e equilibrar o nível de sódio ou consumo de potássio.

Portanto, conclui-se que existem lacunas no conhecimento sobre quais padrões alimentares devem ser adotados para 
conduzir um paciente com DRC. Assim, são necessárias mais pesquisas para abordar esses padrões, uma vez que não se encontram evidências claras e precisas para apoiar em um padrão alimentar, sobretudo nos doentes renais em estágios avançados. Por fim, recomenda-se que o padrão alimentar indicado é aquele que o nutricionista através da sua análise clínica e crítica, com base na história dietética, complicações e/ou progressão da doença, vai adotar para a terapêutica a ser instituída para/com o paciente, visando incrementar a sua qualidade de vida e longevidade.

\section{Referências}

Banerjee, T., liu, Y., \& Crews, D. C. (2016). Dietary Patterns and CKD Progression. Blood Purif. San Francisco. 17 (1), 117-112. https://doi.org/10.1159/000441072

Brzózka, A. C., Franszyk, B., \& Rysz, J. (2017). Vegetarian Diet in Chronic Kidney Disease: A Friend or Foe. Nutrients. Espanha. 2 (1), 1-15. https://doi.org/10.3390/nu9040374.

Cases, A., et al. (2019). Vegetable-Based Diets for Chronic Kidney Disease? It Is Time to Reconsider. Nutrients. Espanha. 11 (1), 1-26. https://doi.org/10.3390/nu11061263.

Carrero, J. J., et al. (2020). Plant-based diets to manage the risks and complications of chronic kidney disease. Nature reviews Nephrology. Mexico. 1 (4), 1 18. https://doi.org/10.1038/s41581-020-0297-2.

Clegg, D. J., \& Gallant, K. M. H. (2019). Plant-Based Diets in CKD. Clinical Journal of the American Society of Nephrology. Minneapolis. 14 (1), 1-3. https://doi.org/10.2215/CJN.08960718.

Chauveau, P., et al (2019). Vegetarian diets and chronic kidney disease. Nephrology Dialysis Transplantation. Bordeaux. 34 (1), $199-207$. https://doi.org/10.1093/ndt/gfy164.

Chauveau, F., et al (2017). Mediterranean diet as the diet of choice for patients with chronic kidney disease. Nephrol Dial Transplant. Canada. 1 (1), 1-11. https://doi.org/10.1093/ndt/gfx085.

Cupisti, A., et al (2017). Non-Traditional Aspects of Renal Diets: Focus on Fiber, Alkali and Vitamin K1 Intake. Nutrients. Espanha. 9 (1), 1-15. https://doi.org/10.3390/nu9050444.

Cuppari, L., (2019). Guia de Nutrição: nutrição clínica no adulto. (4º Ed.). Manole.

Donato, H., \& Donato, M (2019). Etapas na Condução de uma Revisão Sistemática. Acta Med Port. Portugal, Portugual, 32 (3), $227-235$.

Goraya, N., \& Wesson, D. E. (2015). Dietary interventions to improve outcomes in chronic kidney disease. Nephrology and Hypertension. Boston. 24 (4), 505-510. https://doi.org/10.1097 / MNH.0000000000000160.

Ikizler., et al (2020). KDOQI Clinical Practice Guideline for Nutrition in CKD:2020. 76 (4), 1-107. https://doi.org/10.1053/j.ajkd.2020.05.006.

Joshi, S., Mcmacken, M., \& Zadeh, K. K. (2020). Plant-Based Diets for Kidney Disease: A Guide for Clinicians. American Journal of Kidney Diseases. Europen. 1 (1), 1-28. https://doi.org/10.1053/j.ajkd.2020.10.003.

Joshi, S., Shah, S., \& Zadeh, K. K. (2018). Adequacy of Plant-Based Proteins in Chronic Kidney Disease. Journal of Renal Nutrition. Pennsylvania. 1 (1), 1-6. https://doi.org/10.1053/j.jrn.2018.06.006.

Krause, M. V., Mahan, L. K., \& Escott-Stump, S. (2018). Krause. Alimentos, Nutrição e Dietoterapia. (14 Ed.) GEN Guanabara Koogan.

Kim, H., et al (2019). Plant-Based Diets and Incident CKD and Kidney Function. Clinical Journal of the American Society of Nephrology. Minneapolis. 14 (2), 1-10. https://doi.org/10.2215/CJN.12391018.

Kammoun, K. (2017). Diet in chronic kidney disease in a Mediterranean African country. BMC Nephrology. Tunisia. 18 (34), 1-7. https://doi.org/10.1186/s12882-017-0448-2.

Kidney International (2020). KDIGO 2020 Clinical Practice Guideline for Diabetes Management in Chronic Kidney Disease. 98 (4), 1-120. https://doi.org/10.1016/j.kint.2020.06.019.

Liu, H. W., et al (2019). Association of Vegetarian Diet with Chronic Kidney Disease. Nutriens. Espanha. 11 (1), 2-8. https://doi.org/10.3390/nu11020279.

Letourneau, P., et al (2020). Source and Composition in Amino Acid of Dietary Proteins in the Primary Prevention and Treatment of CKD. Nutrients. Espanha. 12 (1), 1-21. https://doi.org/10.3390/nu12123892..

Lanuza, F., et al (2020). Wholegrain Consumption and Risk Factors for Cardiorenal Metabolic Diseases in Chile:A Cross-Sectional Analysis of 2016-2017 Health National Survey. Nutrients. Espanha. 12 (1), 1-12. https://doi.org/10.3390/nu12092815.

Maggo, P. S. C., et al (2017). Dietary interventions for adults with chronic kidney disease (Review). Cochrane Database of Systematic Reviews. Espanha. 3 (1), 1-85. https://doi.org/10.1002/14651858.CD011998.pub2. 
Research, Society and Development, v. 10, n. 6, e28610615891, 2021

(CC BY 4.0) | ISSN 2525-3409 | DOI: http://dx.doi.org/10.33448/rsd-v10i6.15891

Messa, P., (2020). Dietary Habits, Vitamin and Mineral Supplements in Patients with Chronic Kidney Disease (CKD). Nutrients. Espanha. 12 (1), 1-4. https://doi.org/10.3390/nu12123817.

Nishank, J., \& Reilly, R. F. (2014). Effects of dietary interventions on incidence and progression of CKD. Nature Reviews Nephrology. Otago. 1 (1), 1-13. 2014. https://doi.org/10.1038/nrneph.2014.192.

Rysz, J., et al (2017). The Effect of Diet on the Survival of Patients with Chronic Kidney Disease. Nutrients. Espanha. 9 (1), 1-17. https://doi.org/10.3390/nu9050495.

Ramos, C. I., et al (2020). Does dietary potassium intake associate with hyperkalemia in patients with chronic kidney disease? Nephrol Dial Transplant. 28 (1), 1-9. https://pubmed.ncbi.nlm.nih.gov/33247727/.

Serdan, F. P., et al (2020). Nutritional Guideline for the Management of Mexican Patients with CKD and Hyperphosphatemia. Nutrients. Espanha. 12 (1), 120. https://doi.org/10.3390/nu12113289.

Sparks, B. (2018). Nutritional Considerations for Dialysis Vegetarian Patients, Part Two. Journal of Renal Nutrition, United State. 28 (3), 19-23. 2018. https://doi.org/10.1053/j.jrn.2017.12.004. 